\title{
Ureteral stent-related aortoureteric fistula: case report and literature review
}

\author{
Pankaj P Dangle, MD; ${ }^{*}$ Robert Bahnson, $M D ;^{\dagger}$ Ashay Patel, $D 0^{* \dagger}$
}

\begin{abstract}
We describe a case of early postoperative aortoureteric fistula following exenterative surgery treated with wet ostomy with bilateral ureterosigmoidostomies and ureteric stent placement. A high index of clinical suspicion is required, as diagnosis is often difficult.
\end{abstract}

Can Urol Assoc J 2009;3(6):E84-E86

\section{Introduction}

Ureteral stent-related vascular complications are rare and can be potentially fatal. ${ }^{1,2}$ Most complications are common in the setting of previous vascular surgery and/or atherosclerosis. Establishing a diagnosis in complex cases is often difficult; in the presence of hematuria a high index of clinical suspicion is warranted. We describe a case of aortoureteric fistula, presenting as early postoperative hematuria.

\section{Case history}

A 62-year-old male with a recurrent rectal cancer underwent a pelvic exenteration, intraoperative radiotherapy and wet ostomy with bilateral ureterosigmoidostomies and ureteral stent placement (Percuflex Stent, $7 \mathrm{fr}, 80 \mathrm{~cm}$, Boston Scientic, Natick, MA). He had an uneventful recovery. Five weeks after surgery, the patient presented with flank pain and intermittent gross hematuria through and around the stents for several days. On admission, a computerized axial tomography of the abdomen and pelvis revealed bilateral ureteral stents and new bilateral hydronephrosis with hyperdense material in the collecting system consistent with blood. He developed hemodynamic instability due to anemia and had a blood transfusion. Hematuria continued for 24 hours following stent removal. Once stable and hematuria resolved, he underwent angiography, which revealed bilateral renal artery fibromuscular dysplasia and a pseudoanuerysm at the middle branch of the right renal artery (Fig. 1, Part a).
The following day, he underwent exploration of the peritoneal cavity for suspected peritonitis. We found no obvious pathology related to the bowel, urinary or fecal diversion; however, a retroperitoneal hematoma was identified, without an obvious source of bleeding. Bleeding from the gastrointestinal tract was ruled out with endoscopy.

The pseudoanuerysm was embolized on the assumption that it was the source of bleeding, due to recurrent bleeding. After the procedure, the patient remained asymptomatic for a period of 5 days, but developed another episode of bleeding from the urostomy. Bedside pouchoscopy of the urinary limb isolated the bleeding to the right ureteral orifice. The right renal artery was subsequently embolized and he remained asymptomatic for about 10 days (Fig.1, Part b). A massive episode of bleeding was noted from his midline wound into the wound vacuum-assisted closure device, which was left for a superficial wound separation following exploratory laparotomy for peritonitis. This bleeding led to hypovolemic shock and cardiac arrest. After successful revival, the patient had an emergent exploratory laparotomy, which revealed a large retroperitoneal hematoma and no source of intraabdominal bleeding. During the retroperitoneal exploration, the patient developed another cardiac arrest. He was revived and the procedure was abandoned. Following another episode of bleeding 24 hours later, he had repeat angiography (the fourth one since admission), which demonstrated a communication between the distal aortic pseudoanuerysm and the right ureter as it crossed over the aortic bifurcation (Fig. 2). The patient was taken to the operating room emergently to be operated on by a vascular surgeon. Unfortunately, he remained unstable and died. At the family's request, the patient was not subjected to a post-mortem examination.

\section{Discussion}

Aortoureteric fistula has been documented in association with prior pelvic exenteration, radiation and vascular surgery. ${ }^{1,2}$ Ureteral stent-related aortoureteric fistulas are reported in a small number of cases, and mostly in non- 


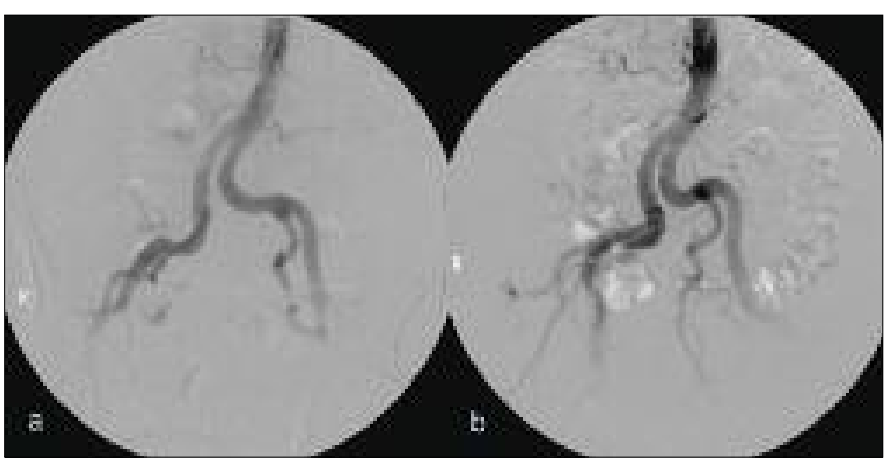

Fig. 1. Angiographic imaging during (a) initial bleeding episode, (b) when right renal artery emobolization failed to demonstrate the fistula.

English literature. The causative factor has been attributed to prolonged stent placement. ${ }^{2-6}$

Most cases report prolonged duration of stent placement varying from 5 months to 15 years before the onset of hematuria. ${ }^{3,4}$ The hematuria is either intermittent or life-threatening, with some patients presenting spontaneously and others during stent exchange. In our case, the patient presented with intermittent hematuria 5 weeks after the initial stent placement, and he remained unstable during hospitalization.

Fistulous communication with the aorta, which is a highpressure system, should theoretically present with continuous bleeding; a $1.5-\mathrm{cm}$ fistula is relatively large for intermittent self-remitting bleeding. Clinical presentation (like the one experienced by our patient) and coexistent vascular anomalies, with inconclusive angiographic imaging, can be misleading criteria in establishing a diagnosis; this situation can result in delayed diagnosis.

A high index of clinical suspicion and angiographic imaging and retrograde study are useful for diagnosis. Pouchoscopy was useful in lateralizing the side of the bleeding. Failure to identify the fistulous communication during the first three angiographic studies can be attributed to the lack of active bleeding during the study. Even the first surgical exploration of the retroperitoneal hematoma failed to reveal the source of bleeding.

The diagnosis of aortoureteric fistula can be elusive and may be delayed depending on the clinical scenario. Standard angiography is diagnostic in $23 \%$ to $41 \%$ of cases; ${ }^{7-9}$ the provocative technique is positive in $63 \%$ of patients, as experienced by Krambeck and colleagues. ${ }^{10}$ In our case, angiography failed to detect the fistula in 3 of the 4 times, with the last angiography identifying the location as a result of active bleeding during the study. Other diagnostic studies, such as retrograde pyelogram and ureteroscopy, add minimal value in the presence of significant bleeding other than lateralizing the source of the hematuria. ${ }^{6}$ One study reported almost $90 \%$ of patients with a correct diagnosis are treated successfully; on the other hand, the mortality for patients with an undiagnosed condition is up to $52 \% .^{5}$
Fig. 2. Angiographic imaging (fourth) demonstrating the fistula between distal aorta and right distal ureter with pseudoanuerysm.

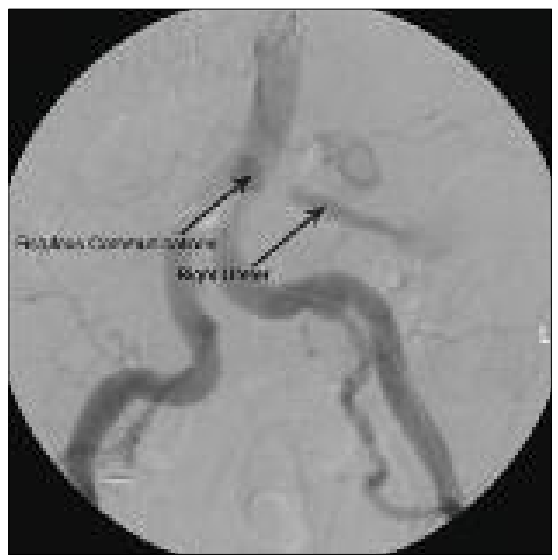

The etiology of aortoureteric fistula is not well understood. A possible mechanism is the theory of a "counterbrace" leading to alteration in the ureteral wall elasticity with eventual pressure necrosis. ${ }^{7}$ The other plausible theory is postoperative inflammatory reaction or associated infection leading to inflammatory ureteral fixation to the anterior wall of aorta leading to erosion. On preoperative imaging, this patient had radiographic signs of vascular disease (atherosclerosis, fibromuscular dysplasia), with skeletonization of the aorta; the course of the ureter may have increased his risk of fistula formation.

Intraoperative radiation therapy was delivered to the left pelvic sidewall to address positive margin measuring about $5 \mathrm{~cm}$. The site of the radiation therapy was away from the site of fistulous communication with the distal aorta and the right ureter, thus making it a less likely etiology for the fistula formation. Fajardo and Lee have reported the major vascular complications associated with, but not clearly attributed to, radiotherapy with high tumour lytic dose and after a prolonged period of time. ${ }^{11}$ A number of factors, such as tumour recurrence, surgery and its complications and abscess, contributed to the rupture of the vessels. ${ }^{11}$

Multiple treatment options, such as open repair with ligation and endovascular stenting, are available with various success rates. ${ }^{6,10}$ Endovascular stenting has been proven to be effective, and provides a faster resolution especially in a setting of massive hemorrhage. ${ }^{3}$

To our knowledge, an early presentation of aortoureteric fistula secondary to ureteral stent has not been reported. Such a possibility could be explained due to the combined effect of high aortic pressure and the counter-brace effect of the stent. An aortoureteric fistula should be suspected in patients with early postoperative significant hematuria, associated clinical signs of acute blood loss in a setting of pelvic exenteration, urinary diversion with ureteric stents and an inconclusive angiographic study. A high index of clinical suspicion, with a low threshold for surgical exploration, and correction of pathology are essential. Apart from the high index of clinical suspicion, using a multidisciplinary approach 
Dangle et al.

that includes an interventional radiologist, a vascular surgeon and a urologist is important in dealing with such a rare complication.

\section{Conclusion}

Ureteric stent-related vascular fistulas are an uncommon scenario, but should be suspected in an early and delayed post-exenterative setting presenting with hematuria. As practising urologists, we should be aware of such a rare, but problematic, complication.

From the * Ohio State University Comprehensive Cancer Center, Columbus, $\mathrm{OH}$; ${ }^{\dagger}$ Department of Urology, The Ohio State University, Columbus $\mathrm{OH}$

Competing interests: None declared.

This paper has been peer-reviewed.

\section{References}

1. Batter SJ, McGovern FJ, Cambria RP. Ureteroarterial fistula: case report and review of the literature. Urology 1996;48:487-9.

2. Ota T, Tsuji Y, Kawasaki R, et al. Endovascular treatment of aortoureteric fistula. J Endovasc Ther. 2005;12(3):411-413

3. Takayama $Y$, Tada $Y$, Takagi A, et al. A successfully treated case of aortouretric fistula associated with pelvic exenteration. Nippon Geka Gakkai Zasshi 1990;91:645-8.

4. Segawa N, Hamada S, Takahara K, et al. Ureteral-abdominal aortic fistula during the exchange of ureteral stenting: a case report. Hinyokika Kiyo 2007;53:565-9.

5. Keller FS, Barton RE, Routh WD, et al. Gross hematuria in two patients with ureteral-ileal conduits and double-J stents. J Vasc Interv Radiol 1990;1:69-77.

6. Madoff DC, Gupta $S$, Toombs BD, et al. Arterioureteral fistulas: a clinical, diagnostic, and therapeutic dilemma. AJR Am J Roentgenol 2004;182:1241-50.

7. Devonian $P$, Castaigne D, Travagli JP, et al. Arterioureteral fistula after extended resection of pelvic tumors: report of three cases and review of the literature. Ann Vasc Surg 1992;6:362-9.

8. Vandersteen DR, Saxon RR, Fuchs E, et al. Diagnosis and management of ureteroiliac artery fistula: value of provocative arteriography followed by common iliac artery emobolization and extra anatomic arterial bypass grafting. J Urol 1997; 158:754-8.

9. Quillin SP, Darcy MD, Picus D. Angiographic evaluation and therapy of ureteroarterial fistula. Am J Radiol 1994;162:873.

10. Krambeck AE, DiMarco DS, Gettman MT, et al. Ureteroiliac artery fistula: diagnosis and treatment algorithm. Urology 2005;66:990-4.

11. Fajardo LF, Lee A. Rupture of major vessels after radiation. Cancer 1975;36:904-13.

Correspondence: Dr. Pankai P. Dangle, The Ohio State University, Comprehensive Cancer Center, 538 Doan Hall, 410 West 10th Ave, Columbus, Ohio, 43210; fax: 614-293-0982; Pankai.Dangle@osumc.edu 